\title{
Temperature Renormalization in the Non Linear Vlasov Problem
}

\author{
W. L. Sadowski and Z. G. Ruthberg \\ Institute for Basic Standards, National Bureau of Standards, Washington, D. C. 20234
}

(August 22, 1969)

\begin{abstract}
Ways of improving the representation of the velocity distribution function in the solution of the non-linear Vlasov equation are discussed. Hermite polynomial coefficients for temperature renormalization are derived. Transformation properties of Hermite polynomials necessary to do this are discussed. Analytic expressions for the truncation error of the temperature renormalized functions are compared with the computer results.
\end{abstract}

Key words: Expansion coefficients; Hermite polynomials; nonlinear; numerical; plasma; truncation error; Vlasov equation.

\section{Introduction}

This paper deals with some mathematical aspects of the numerical solution of the non-linear Vlasov equation by means of eigenfunction expansions $[1,2,3] . .^{1}$ The model studied is a onedimensional, electrically neutral plasma undergoing longitudinal oscillations. Periodic boundary conditions are imposed on the electron density. The initial distribution function is given in dimensionless form [8] by the expression,

$$
f(x, v, t=0)=(1+\alpha \cos k x) e^{-v^{2} / 2} / \sqrt{2 \pi}
$$

where $\alpha$ is the non-linearity parameter and $k=2 \pi / L, L$ being the periodic length. The electric field is given by the Poisson equation and has the form

$$
E(x, t)=\int_{0}^{x} d x^{\prime} \int_{-\infty}^{+\infty} f\left(x^{\prime}, v, t\right) d v-x
$$

The initial value of the electric field is thus $E(x, t=0)=(\alpha / k) \sin k x$. The ratio $(\alpha / k)$ determines the initial field amplitude and the initial potential energy content of the system.

In applying the eigenfunction expansion method to the numerical solution of nonlinear partial differential equations, it is very important to obtain as much analytic information as possible concerning expansion properties of the solution, the truncation error incurred by neglecting all but a finite number of terms in the expansion and the effect of truncation errors on the validity of the solution. Procedures of questionable validity are those in which the solution is expanded into a set of orthogonal polynomials without some knowledge of convergence properties of the expansion. When there is no proof that the solution exists on the interval over which the equation is being integrated, extreme caution should be exercised in evaluating numerical results. The lack of general methods of dealing with nonlinear partial differential equations makes one's intuition an unreliable guide in judging numerical results. 
Truncation of an expansion with time-dependent coefficients is a particularly difficult problem since the function that was well represented initially by a certain expansion may no longer be adequately represented by the same number of terms at a later time. The usual numerical tests employed in finite difference methods, such as conservation of certain time-independent quantities, may be inapplicable or not very significant. The physically observable quantities like the number density and macroscopic currents are determined by the first few moments of the distribution function of the phase space coordinates, $f(x, v, t)$. In this sense, the first few moments of an eigenfunction expansion are fixed [4] since they must reproduce the observed quantities. For example, conservation of density in phase space and of total energy in the nonlinear Vlasov equation puts conditions on the zeroth, first, and second moments of the Hermite polynomial expansion. Of these, the zeroth is automatically conserved, while the other two do not indicate any trouble until long after the numerical solution has become invalid.

Associated with the question of truncation is the problem of finding a transformation to a different set of eigenfunctions in the course of the calculation which would give a better representation of the function as it evolves with time than the initial set of eigenfunctions. If such a transformation could be automatically found the truncation error would be reduced. This is the problem discussed below.

In section 1 is derived a transformation of the arguments of Hermite polynomials with the aim of reducing the truncation error with a fixed number of terms in the expansion. The transformation is applied to the problem of temperature renormalization in section 2 and the expansion coefficients are explicitly given.

In section 3 error bounds are derived for the truncation and it is shown that for $\frac{\alpha}{k} \leqslant 1$ temperature renormalization does not result in a significant reduction of truncation error.

Comparison of analytic results with those obtained on the computer is given in appendix II.

\section{Transformation Properties of Hermite Polynomials}

This section deals with certain transformation properties of Hermite polynomials. These properties will be used in evaluating expansion coefficients of the two-stream distribution function and in temperature normalization. A few necessary formulas pertaining to Hermite polynomials [5] will be given in eqs (1.1), (1.2), and (1.3). Hermite polynomials ${ }^{2}$ are defined by means of the relation

$$
e^{x t-t^{2} / 2}=\sum_{n=0}^{\infty} \frac{H e_{n}(x) t^{n}}{n !} .
$$

From eq (1.1) it follows that

$$
H e_{n}(x)=\sum_{k=0}^{[n / 2]} \frac{(-1)^{k} n !}{2^{k} k !(n-2 k) !} x^{n-2 k}
$$

where $[n / 2]$ is the greatest integer.

From the same equation the following relation can be derived

$$
x^{n}=\sum_{k=0}^{[n / 2]} \frac{n !}{2^{k} k !(n-2 k) !} H e_{n-2 k}(x) .
$$

\footnotetext{
${ }^{2}$ For discussion of differences in notation and generating functions see appendix I.
} 


\subsection{Scale Transformation}

Given a Hermite polynomial, $H e_{n}\left(\frac{v}{\sqrt{\epsilon}}\right)$, we would like to express it in terms of $\sum_{m} H e_{m}(v)$. Using eq (1.2) we obtain

$$
\begin{aligned}
H e_{n}\left(\frac{v}{\sqrt{\epsilon}}\right) & =\sum_{k=0}^{[n / 2]} \frac{(-1)^{k} n !}{2^{k} k !(n-2 k) !} \quad \frac{v^{n-2 k}}{\epsilon^{(n-2 k) / 2}} \\
& =\frac{1}{\epsilon^{n / 2}} \sum_{k=0}^{[n / 2]} \frac{(-1)^{k} n ! \epsilon^{k}}{2^{k} k !(n-2 k) !} v^{n-2 k} .
\end{aligned}
$$

By eq (1.3) we have:

$$
H e_{n}\left(\frac{v}{\sqrt{\epsilon}}\right)=\frac{1}{\epsilon^{n / 2}} \sum_{k=0}^{[n / 2]} \frac{(-1)^{k} n ! \epsilon^{k}}{2^{k} k !(n-2 k) !} \sum_{l=0}^{[(n-2 k) / 2]} \frac{(n-2 k) !}{2^{l} l !(n-2 k-2 l) !} H_{n-2 k-2 l}(v) .
$$

Let $k+l=m$. We obtain

$$
H e_{n}\left(\frac{v}{\sqrt{\epsilon}}\right)=\frac{1}{\epsilon^{n / 2}} \sum_{m=0}^{[n / 2\rfloor} \sum_{k=0}^{m} \frac{(-1)^{k} \epsilon^{k} n !}{2^{m} k !(m-k) !(n-2 m) !} H e_{n-2 m}(v) .
$$

But the summation on $k$ is a binomial expansion of $\frac{1}{m !}(1-\epsilon)^{m}$.

This leads to the final expression

$$
H e_{n}\left(\frac{v}{\sqrt{\epsilon}}\right)=\frac{1}{\epsilon^{n / 2}} \sum_{m=0}^{[n / 2]} \frac{n !(1-\epsilon)^{m}}{2^{m} m !(n-2 m) !} H e_{n-2 m}(v) .
$$

which for $\epsilon=1$ yields the required identity

$$
H e_{n}\left(\frac{v}{\sqrt{\epsilon}}\right)=H e_{n}(v), \quad \epsilon=1
$$

\subsection{Change of Origin}

Given a Hermite polynomial, $H e_{n}\left(\frac{v-a}{\sqrt{\epsilon}}\right)$, we shall express it in terms of $\sum_{m} H e_{m}(v)$.

Let

$$
v^{\prime}=\frac{v}{\sqrt{\epsilon}} \quad \text { and } \quad a^{\prime}=\frac{a}{\sqrt{\epsilon}}
$$

From eq (1.2) we have

$$
\begin{aligned}
H e_{n}\left(v^{\prime}-a^{\prime}\right) & =\sum_{k=0}^{[n / 2]} \frac{(-1)^{k} n !}{2^{k} k !(n-2 k) !}\left(v^{\prime}-a^{\prime}\right)^{n-2 k} \\
& =\sum_{k=0}^{[n / 2]} \sum_{m=0}^{n-2 k} \frac{(-1)^{k} n !}{2^{k} k ! m !(n-2 k-m) !} v^{n-2 k-m}\left(-a^{\prime}\right)^{m} .
\end{aligned}
$$


Summation indices in the nested double sum in eq (1.9) can be interchanged to give the following expression:

$$
H e_{n}\left(v^{\prime}-a^{\prime}\right)=\sum_{m=0}^{n} \sum_{k=0}^{[(n-m) / 2]} \frac{(-1)^{k}(n-m) !}{2^{k} k !(n-m-2 k) !} v^{\prime n-m-2 k} \frac{n !\left(-a^{\prime}\right)^{m}}{m !(n-m) !}
$$

Using eq (1.2) with $n$ replaced by $n-m$ we obtain

$$
H e_{n}\left(v^{\prime}-a^{\prime}\right)=\sum_{m=0}^{n} H e_{n-m}\left(v^{\prime}\right) \frac{n !\left(-a^{\prime}\right)^{m}}{m !(n-m) !} .
$$

Similarly, we have

$$
H e_{n}\left(v^{\prime}+a^{\prime}\right)=\sum_{m=0}^{n} H e_{n-m}\left(v^{\prime}\right) \frac{n ! a^{\prime m}}{m !(n-m) !} .
$$

Substituting $\frac{v}{\sqrt{\epsilon}}$ for $v^{\prime}$ and $\frac{a}{\sqrt{ } \epsilon}$ for $a^{\prime}$ we get with the aid of eq (1.7)

$$
H e_{n}\left(\frac{v-a}{\sqrt{\epsilon}}\right)=\sum_{m=0}^{n} \frac{n !(-a)^{m} \epsilon^{-m / 2}}{m !(n-m) !} \frac{1}{\epsilon^{(n-m) / 2}} \sum_{k=0}^{[(n-m) / 2]} \frac{(n-m) !(1-\epsilon)^{k}}{2^{k} k !(n-m-2 k) !} H e_{n-m-2 k}(v) .
$$

Let $m+2 k=p$

$$
\begin{aligned}
H e_{n}\left(\frac{v-a}{\sqrt{\epsilon}}\right) & =\frac{1}{\epsilon^{n / 2}} \sum_{p=0}^{n} \sum_{k=0}^{[p / 2]} \frac{n ! a^{p-2 k}(-1)^{p-2 k}(1-\epsilon)^{2 k / 2}}{(p-2 k) ! 2^{k} k !(n-p) !} H e_{n-p}(v) \\
& =\frac{1}{\epsilon^{n / 2}} \sum_{p=0}^{n} \frac{n !(\epsilon-1)^{p / 2}}{p !(n-p) !} H e_{n-p}(v) \sum_{k=0}^{[p / 2]} \frac{(-1)^{k} p !\left(\frac{-a}{\sqrt{\epsilon-1}}\right)^{p-2 k}}{2^{k} k !(p-2 k) !} .
\end{aligned}
$$

The last sum in eq (1.13) is a Hermite polynomial of the argument $\frac{-a}{\sqrt{\epsilon-1}}$ and we obtain:

$$
H e_{n}\left(\frac{v-a}{\sqrt{\epsilon}}\right)=\frac{1}{\epsilon^{n / 2}} \sum_{p=0}^{n} \frac{n !(\epsilon-1)^{p / 2}}{p !(n-p) !} H e_{n-p}(v) H e_{p}\left(\frac{-a}{\sqrt{\epsilon-1}}\right) .
$$

Similarly, we have

$$
H e_{n}\left(\frac{v+a}{\sqrt{\epsilon}}\right)=\frac{1}{\epsilon^{n / 2}} \sum_{p=0}^{n} \frac{n !(\epsilon-1)^{p / 2}}{p !(n-p) !} H e_{n-p}(v) H e_{p}\left(\frac{a}{\sqrt{\epsilon-1}}\right) .
$$

With (1.14) and (1.15) we find, as we must, that in the limit $a=0$ they reduce to $(1 \cdot 7)$ and for $\epsilon=1$ they give eqs (1.10) and (1.11) respectively. It should be noted that imaginary values of $\sqrt{\epsilon-1}$ for $\epsilon<1$ in the argument of $\mathrm{He}_{p}$ are immaterial to the analysis since the product $(\epsilon-1)^{p / 2} \mathrm{He}_{\underline{p}}$ $\left(\frac{a}{\sqrt{\epsilon-1}}\right)$ is always real. This can be seen by expressing $H e_{p}$ as in eq (1.1). 


\section{Temperature Renormalization}

In the course of time the initial potential energy of a collisionless plasma is converted into kinetic energy by the Landau damping [6] mechanism. The velocity distribution function initially given by [1]

$$
f(v, t=0)=\frac{1}{\sqrt{2 \pi}} H e_{0}^{-v^{2} / 2}
$$

will change to reflect the increase in kinetic energy. The wings of the function will rise and the rise will be given by the set of Hermite polynomials $\left\{C_{n} H e_{n}(v)\right\}$. It must be noted that since no collisions are present, the system will not be in thermal equilibrium at $t \neq 0$ and consequently cannot be represented as it was initially, as a product of a Maxwellian and $\mathrm{He}_{0}$. An exponential which decreases less rapidly with increasing $v$ may however represent the velocity distribution function better than the set $\left\{C_{n} H e_{n}(v) e^{-v^{2} / 2}\right\}$. We shall let

$$
f(v, t)=\frac{1}{\sqrt{2 \pi}} \sum_{n} \bar{C}_{n}(t) \mid \boldsymbol{H} e_{n}(v) e^{-v^{2} / 2 \epsilon(t)}
$$

where the value of $\epsilon(t)$ is determined by the increase in kinetic energy at the time $t$ and $\bar{C}_{n}$ is the set of coefficients associated with the new envelope function $e^{-v^{2} / 2 \epsilon(t)}$. The new representation may be more rapidly convergent and hence require fewer terms for a given truncation error, thus reducing the computer time, or generate a smaller truncation error for the same number of terms.

We shall give below a transformation which will permit the use of the modified exponential of eq (2.2) for the envelope of the velocity distribution function. Let us assume that the velocity distribution function, at a given time $t$, is given by the expressions

$$
\begin{aligned}
& f(v, t)=\sum_{m=0}^{M} C_{m}(t) H e_{m}(v) e^{-\frac{v^{2}}{2 \epsilon(\tau)}} \\
& f(v, t)=\sum_{n=0}^{N} \bar{C}_{n}(t) H e_{n}(v) e^{-\frac{v^{2}}{2 \epsilon(t)}}
\end{aligned}
$$

where $\epsilon(\tau)$ refers to the value of $\epsilon$ calculated at some previous time $\tau$ and $\epsilon(t)$ reflects the current increase in kinetic energy. Thus we get

$$
\bar{C}_{n}=\frac{1}{\sqrt{2 \pi} n} \sum_{m=0}^{M} C_{m}(t) \int_{-\infty}^{\infty} H e_{n}(v) H e_{m}(v) e^{-\frac{v^{2}}{2 \epsilon(\tau)}+\frac{v^{2}}{2 \epsilon(t)}-\frac{v^{2}}{2}} d v
$$

Let

$$
\frac{\epsilon(\tau) \epsilon(t)+\epsilon(t)-\epsilon(\tau)}{\epsilon(\tau) \epsilon(t)}=\gamma \text { and }
$$

let

$$
v \sqrt{\gamma}=v^{\prime} .
$$

Then

$$
\bar{C}_{n}(t)=\frac{1}{\sqrt{2 \pi} n !} \frac{1}{\sqrt{\gamma}} \sum_{m=0}^{M} C_{m} \int_{-\infty}^{\infty} H e_{n}\left(\frac{v^{\prime}}{\sqrt{\gamma}}\right) H e_{m}\left(\frac{v^{\prime}}{\sqrt{\gamma}}\right) e^{-v^{\prime 2} / 2} d v^{\prime}
$$

and conversely we have

$$
C_{m}(t)=\frac{1}{\sqrt{2 \pi} m ! \cdot \sqrt{\beta}} \sum_{n=0}^{N} \bar{C}_{n}(t) \int_{-\infty}^{\infty} H e_{n}\left(\frac{v^{\prime}}{\sqrt{\beta}}\right) H e_{m}\left(\frac{v^{\prime}}{\sqrt{\beta}}\right) e^{-v^{\prime 2} / 2} d v^{\prime}
$$


In the above equation

and

$$
\beta=\frac{\epsilon(t) \epsilon(\tau)+\epsilon(\tau)-\epsilon(t)}{\epsilon(t) \epsilon(\tau)}
$$

$$
v \sqrt{\beta}=v^{\prime} .
$$

Let us assume that the $C_{m}$ are known. Using eq (1.7) to express $H e_{n}\left(\frac{v^{\prime}}{\sqrt{\gamma}}\right)$ in terms of $H e_{n}\left(v^{\prime}\right)$ we obtain with the use of orthogonality conditions the following expression for $\bar{C}_{n}(t)$ :

$$
\bar{C}_{n}(t)=\frac{1}{\sqrt{\gamma}} \sum_{m=0}^{M} \sum_{k=0}^{[m / 2]} \sum_{l=0}^{[n / 2]} C_{m}(t) \frac{1}{\gamma^{(m+n) / 2}} \frac{m !(1-\gamma)^{k+l}}{2^{k+l} k !(m-2 k) ! l !(n-2 l) !} \delta_{n-2 l, m-2 k} .
$$

The orthogonality condition requires that $2 k=m-n+2 l$ and eq (2.7) reduces to

$$
\bar{C}_{n}(t)=\frac{1}{\sqrt{\gamma}} \sum_{m=0}^{M} C_{m}(t) \sum_{l=0}^{[n / 2]} \frac{m !(1-\gamma)^{[(m-n) / 2]+2 l}}{\gamma^{(m+n) / 2} 2^{(m-n+4 l) / 2}((m-n+2 l) / 2) !((n-2 l) !)^{2} l !}
$$

with the negative factorial being defined as zero. The double sum in eq (2.8) can be written in a different form in which no negative factorials occur:

$$
\bar{C}_{n}(t)=\frac{1}{\sqrt{\gamma}} \sum_{l=0}^{[n / 2]} \sum_{m=n-2 l}^{M} C_{m}(t) \frac{m !(1-\gamma)^{[(m-n) / 2]+2 l}}{\gamma^{(m+n) / 2} 2^{(m-n+4 l) / 2}((m-n+2 l) / 2) !((n-2 l) !)^{2} l !}
$$

It is very difficult to make any comparison between $\bar{C}_{n}$ and $C_{m}$ without the knowledge of the actual values of $C_{m}$. Although a bound can be given for $\bar{C}_{n}$ in terms of $C_{0}$ under the assumption that the $C_{m}$ are monotonically decreasing with increasing $m$, this bound is of no practical value because it is too generous.

We shall now turn to the simpler problem of calculating the coefficients in the expansion

In this case we have

$$
e^{-v^{2} / 2 \epsilon}=\sum_{n=0}^{\infty} \bar{C}_{w} H e_{n}(v) e^{-v^{2} / 2}
$$

$$
\epsilon(t)=1 ; \quad \epsilon(\tau)=\epsilon ; \quad \gamma=\frac{1}{\epsilon}
$$

and

$$
C_{0}=1 ; \quad C_{m}=0, \quad m \neq 0 .
$$

Thus the result in eq (2.9) applies to this case for the values of $\gamma$ and $C$ given above. Because the functions in eq (2.10) are symmetric in $v$, only Hermite polynomials with even indices will be present in the expansion. Substituting the values of $\gamma$ and $C_{m}$ into eq (2.9) we obtain:

$$
\bar{C}_{2 q}=\frac{1}{\sqrt{\gamma}} \frac{1}{\gamma^{q}} \frac{(1-\gamma)^{q}}{2^{q} q !}
$$




\section{Error Bounds for the Expansion}

We can obtain an error bound on the truncation of the expansion after the first $N$ terms. Using the asymptotic expression for Hermite polynomials [7] (see appendix I, eq (I.6)) we obtain:

$$
\bar{C}_{2 q} H e_{2 q}(v) \sim \frac{e^{v^{2} / 4} \Gamma(2 q+1)}{2^{q} \Gamma(q+1)} \frac{\sqrt{\epsilon}}{q !}\left(\frac{\epsilon-1}{2}\right)^{q} .
$$

Using Stirling's formula eq (3.1) reduces to

$$
\bar{C}_{2 q} i H e_{2 q}(v) \sim \sqrt{\frac{\epsilon}{\pi}} e^{v^{2 / 4}} \frac{(\epsilon-1)^{q}}{q^{1 / 2}} .
$$

The truncation error is given by the following expression:

$$
R_{N+1} \cong \sum_{p=0}^{\infty} \sqrt{\frac{\epsilon}{\pi}} e^{v^{2 / 4}} \frac{(\epsilon-1)^{N+1+p}}{(N+1+p)^{1 / 2}}
$$

We know that

$$
\frac{(\epsilon-1)^{N+1+p}}{(N+1+p)^{1 / 2}} \leqslant \frac{(\epsilon-1)^{N+1+p}}{(N+1)^{1 / 2}}
$$

for all values of $p$ in eq (3.3) because $\epsilon>1$.

$$
R_{N+1}<\sqrt{\frac{\epsilon}{\pi}} e^{v^{2} / 4} \frac{(\epsilon-1)^{N+1}}{(N+1)^{1 / 2}(2-\epsilon)}
$$

where the summation over $p$ in eq (3.3) has been performed using (3.4).

Let us now estimate a representative value of $\epsilon$ that may occur in the solution of non-linear Vlasov equation. From the introduction we recall that the initial distribution function is given by the expression

$$
f(x, v, t=0)=(1+\alpha \cos k x) e^{-v^{2} / 2} / \sqrt{2 \pi}
$$

where $k=2 \pi / L$ and $L$ is the length of the system. The initial kinetic energy content of the system is given by the integral

$$
K=\int_{0}^{L} d x \int_{-\infty}^{\infty} v^{2} f(x, v, t=0) d v=L
$$

The initial potential energy content is given by

$$
P=\int_{0}^{L} E^{2}(x) d x=\int_{0}^{L} \frac{\alpha^{2}}{k^{2}} \sin ^{2} k x d x
$$

In eq (3.8) the electric field was obtained from eq (3.7) with the aid of Poisson's equation in dimensionless form. Integrating eq (3.8) we obtain the initial potential energy content

$$
P=\frac{\alpha^{2}}{2 k^{2}} L
$$




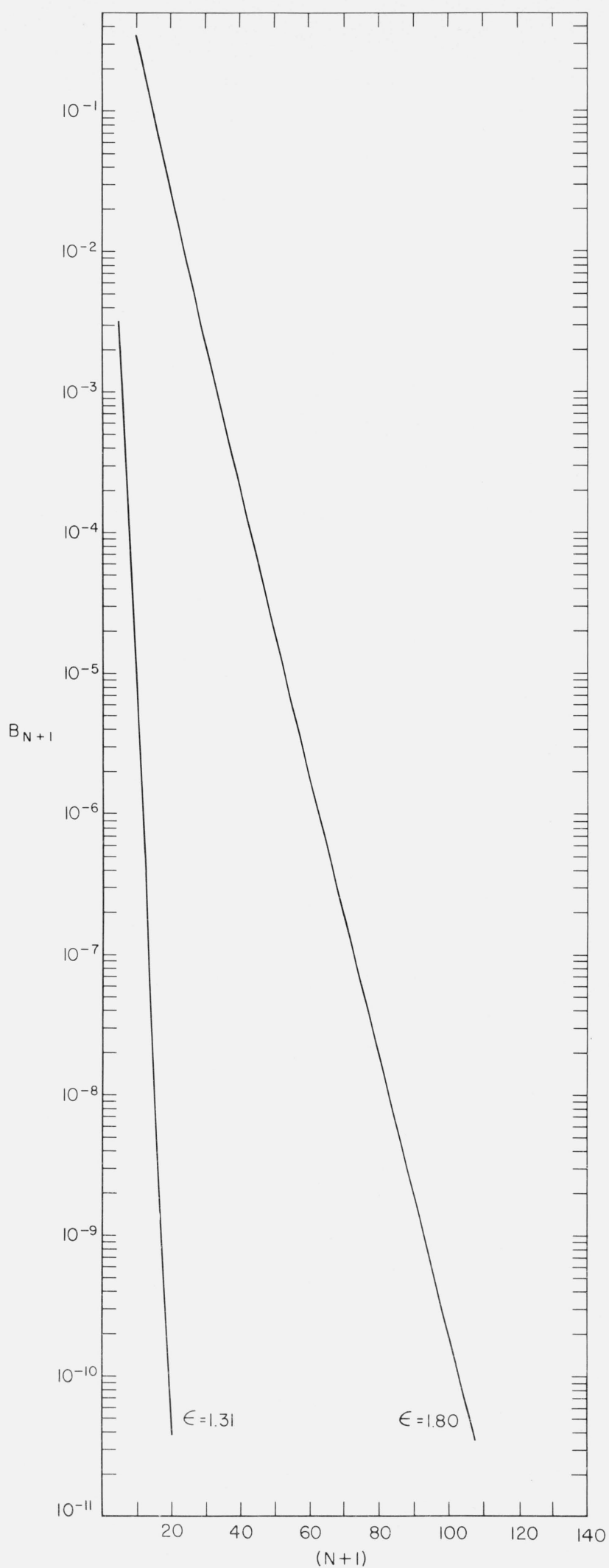
FiguRE 1. Truncation error bound $\mathrm{B}_{\mathrm{N}+1}$ versus $(\mathrm{N}+1)$ for the function $\mathrm{e}^{-\mathrm{v} 2 / 2 \epsilon}=\sum_{\mathrm{n}=0}^{N} \mathrm{C}_{2 \mathrm{n}} \mid \mathrm{He}_{2 \mathrm{n}}(\mathrm{v})$ for $\mathrm{v}=2.0$,
$\epsilon=1.31$ and $\epsilon=1.80$. 
Choosing $\alpha=0.2$ and $k=0.2$ we obtain

$$
P=0.5 L
$$

After the electric field has been completely damped out and the initial potential energy of the field converted to kinetic energy, $\epsilon$ can be calculated from the equation

$$
1.5=\frac{1}{\sqrt{2 \pi}} \int_{-\infty}^{\infty} v^{2} e^{-v^{2} / 2 \epsilon} d v
$$

For the values of $\alpha$ and $k$ given above we obtain the value of $\epsilon$

$$
\epsilon=1.31037 \text {. }
$$

Figure 1 is a plot of the bound $B_{N+1}$ on the truncation error, $R_{N+1}$, as a function of the number of terms, $N$, for $v=2.0, \epsilon=1.31$. Figure 2 is a comparison of the number of terms necessary to represent the function given in eq (2.10) with a truncation error $R_{N+1}<10^{-5} T$ obtained analytically and numerically (see appendix II). The value of velocity chosen for the graph is $v=2$. As can be seen by comparing the analytic and the computer curves, the values of $\epsilon$ close to 1 are in error because the asymptotic form of Hermite polynomials does not apply. One thing that can be concluded from the graphs, however, is that the truncation error cannot be reduced appreciably by reexpressing the solution of the Vlasov equation by a function of the type given in eq $(2.10)$ for the ratio $\alpha / k \leqslant 1$. For ratios $\alpha / k>1$, the potential energy content of the system will lead to a larger increase in kinetic energy as the electric field is damped and a transformation of the type discussed would result in a better representation of the solution.

\section{Appendix I}

There are two different kinds of Hermite polynomials in the literature. One of these is based on the generating function

$$
e^{2 x t-t^{2}}=\sum_{n=0}^{\infty} \frac{H_{n}(x) t^{n}}{n !}
$$

and the other, used in this paper, is usually designated in the literature by He [6]. It is based on the generating function

$$
e^{x t-t^{2} / 2}=\sum_{n=0}^{\infty} \frac{H e_{n}(x) t^{n}}{n !}
$$

The asymptotic expression in the literature [7] is given in the form

$$
\Gamma\left(\frac{1}{2} n+1\right) \exp \left(-\frac{1}{2} x^{2}\right) H_{n}(x)=\Gamma(n+1)\left[\cos \left(N^{1 / 2} x-\frac{1}{2} n \pi\right)+0\left(n^{-1 / 2}\right)\right]
$$

where $N=2 n+1$.

There is a simple transformation that connects the two polynomials. Let

$$
t=\frac{t^{\prime}}{\sqrt{2}} \text { and } x=\frac{x^{\prime}}{\sqrt{2}} .
$$


In eq (I.1) we obtain:

$$
e^{x^{\prime} t^{\prime}-t^{\prime 2} / 2}=\sum_{n=0}^{\infty} \frac{H_{n}\left(\frac{x^{\prime}}{\sqrt{2}}\right) t^{\prime n}}{2^{n / 2} n !}=\sum_{n=0}^{\infty} \frac{H e_{n}\left(x^{\prime}\right) t^{\prime n}}{n !} .
$$

The last equality follows from eq. (I.2).

Using the transformed variable of eq (I.5), the asymptotic form for $\mathrm{He}_{n}(x)$ is

$$
H e_{n}(x)=\frac{e^{x^{2 / 4}}}{2^{n / 2}} \frac{\Gamma(n+1)}{\Gamma\left(\frac{1}{2} n+1\right)}\left[\cos \left(N^{1 / 2} \frac{x}{\sqrt{2}}-\frac{1}{2} n \pi\right)+0\left(n^{-1 / 2}\right)\right]
$$

where again $N=2 n+1$.

\section{Appendix II}

A computer program was written in Fortran to calculate the terms of the expansion of $f(v, t)$ of eq (2.10). The $H e_{2 n}(v)$ and $C_{2 n}$ were calculated recursively. Scaled polynomials, $H e_{2 n}$, were used to stay within the range of the computer in single precision.

$$
\begin{gathered}
\bar{H} e_{2 n}(v)=\frac{1}{\sqrt{(2 n) !}} H e_{2 n}(v) \\
\bar{C}_{2 n}=\sqrt{(2 n) !} C_{2 n} .
\end{gathered}
$$

A partial sum, $P_{n}$, of the first $n$ terms was formed and more terms were added until the relative error ratio test of eq (II.2) was satisfied for one hundred successive terms.

$$
\mid \text { R.E. }\left(P_{n}\right) \mid=\frac{\left|P_{n+1}-P_{n}\right|}{\left|P_{n}\right|}<10^{-5} \text {. }
$$

Using eqs (3.2), (3.5), and (II.2) the three term inequality relating $R_{N+1}$ to the truncation error bound $10^{-5}$ is:

$$
\begin{gathered}
R_{N+1}<\frac{\sqrt{\frac{\epsilon}{\pi}} e^{v^{2} / 4}(\epsilon-1)^{N+1}}{\sqrt{N+1}(2-\epsilon)}<10^{-5} T \\
T=\frac{\sqrt{N}(\epsilon-1)}{\sqrt{N+1}(2-\epsilon)} f e^{v^{2} / 2}
\end{gathered}
$$

where $f$ is the function of eq (2.10). The parameter values investigated were

$$
\begin{aligned}
& \epsilon=1.10,1.20,1.40,1.60,1.80 \\
& v=0.00 \text { to } 6.00 \text { in intervals of } 0.25 .
\end{aligned}
$$

Figure 2 contains a computer curve for $v=2.0$ and a comparison curve based on eq (II.3).

It should be noted that a uniform bound on the truncation error can be obtained by allowing $v$ to assume its largest value. 


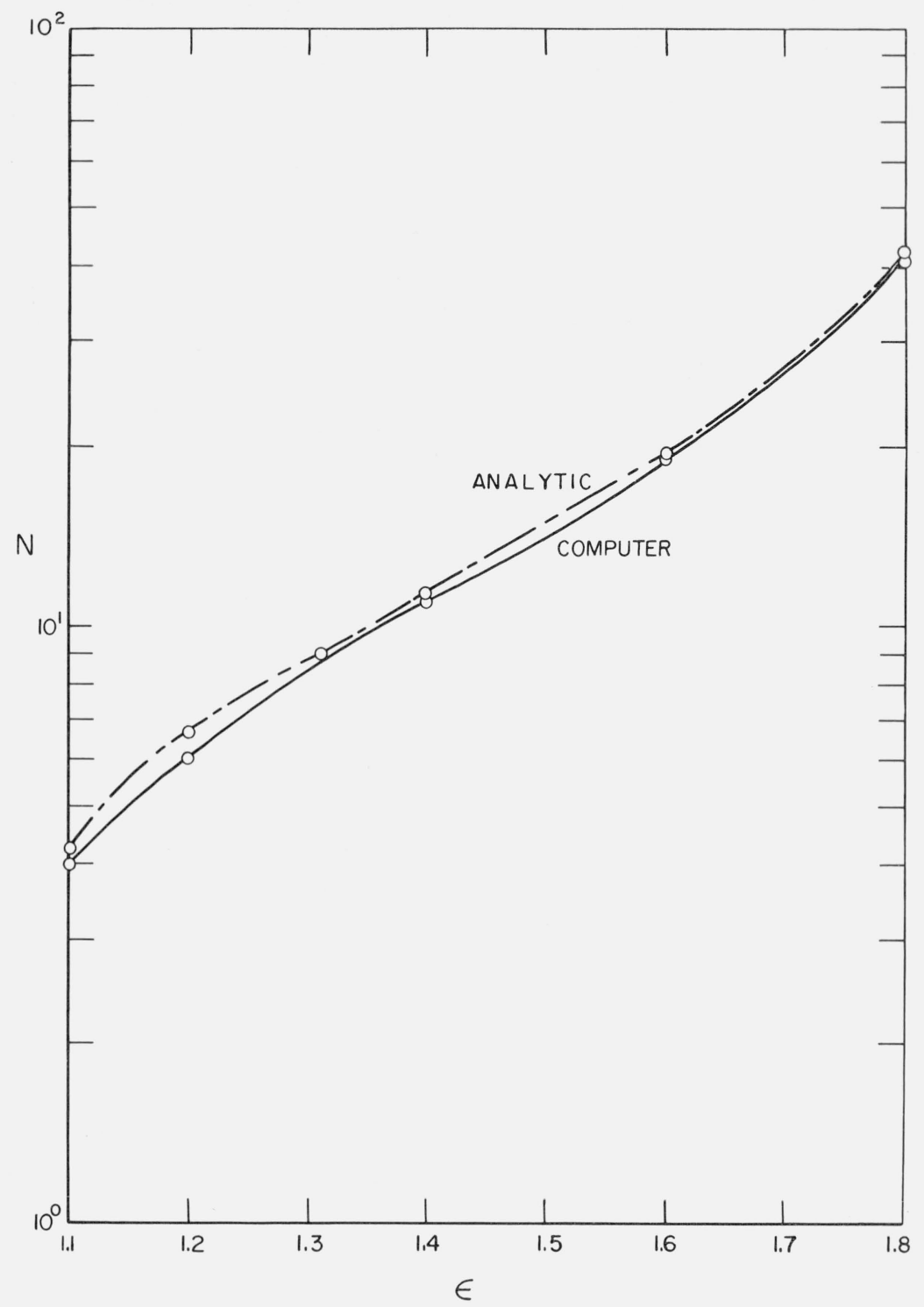

FIGURE 2. $\mathrm{N}$ versus $\epsilon$ for $\mathrm{v}=2.0$ for temperature renormalization curve.

\section{References}

[1] Sadowski, W. L., On Some Aspects of the Eigenfunction Expansion of the Solution of the Non-Linear Vlasov Equation, Symposium on Computer Simulation of Plasma and Many-Body Problems, NASA, SP-153, 433-440, (1967).

[2] Armstrong, T. P., Numerical Studies of the Non-Linear Vlasov Equation, Ph. D. Thesis, Univ. of Iowa, 66-34 (1966).

[3] Feix, M., Engleman, F., Minardi, E., and Oxenius, J., Non-linear Effects from Vlasov's Equation, Phys. Fluids, 6, 266-275, (1963).

[4] Grad, H., On the Kinetic Theory of Rarefied Gases, Comm. Pure and Appl. Math. 2, 331-407 (1949).

[5] Rainville, E. D., Special Functions, 187-194 (Macmillan Co., N.Y., 1960).

[6] Dawson, J., On Landau Damping, Phys. Fluids 4, 869-874 (1961).

[7] Bateman Manuscript Project, Cal. Inst. Technol., Higher Transcendental Functions, A. Erdelyi, editor, Vol. 2, 201 (McGraw-Hill Book Co., N. Y., 1953).

[8] Knorr, G., Numerische Integration der Nichtlinearen Vlasov Gleichung, Z. Naturforsch. 16a, 1320-1328 (1961).

(Paper 73B4-307) 